\title{
Memory of Familiar Melodies: Comparison Between Original and Transposed Keys*
}

\author{
Sumi Shigeno \\ Aoyama Gakuin University, Tokyo, Japan
}

\begin{abstract}
The long-term memory for musical keys of familiar melodies was investigated. An experiment was conducted focusing on memory strength, music familiarity, and key transposition using musical pieces. Participants were eighty-one Japanese undergraduate and graduate students. Eight were absolute pitch (AP) possessors and seventy-three were non-AP possessors. Two pieces of well-known classical music were selected as stimuli. These pieces were played in seven different keys: One was an original key and the other six were transposed keys in which the linear distance and harmonic distance were varied. Participants rated their strength of long-term memory for a particular segment of well-known music by comparing it with their memory of this piece. Importantly they were not required to identify the musical key of the melody. Results indicated that the strength of memory for these musical segments depended mainly on the pitch range associated with the transposed piece and partially on its key. We discussed participants' memory of melodies in the light of linear distance between original and transposed keys, harmonic distance between these factors, and the possibility of absolute tonality.
\end{abstract}

Keywords: musical key, memory of melodies, absolute tonality, transposition, long-term memory

\section{Introduction}

In our daily lives we often listen to music and then sometimes recall them later. Occasionally, music evokes various emotions and physical responses. Tonality, which supports a musical key in western music, is considered to be one of the essential factors that govern our impression of music (Butler \& Brown, 1994) and govern our interpretation of pitches and chords (Temperley \& Marvin, 2008). Key context influences the memory and recognition of melodies (Marvin, 1997). Most composers seem to use the tone-chromatic harmony to express emotions through music; for example, joyful, bored, or peaceful feelings may be generated by listening to tonal music, whereas angry emotions could be caused by atonal music (Thompson \& Robitaille, 1992).

Corso (1957) tested college students majoring in music to determine the accuracy of identifying the equally-tempered tonality (key name and mode). The stimuli consisted of scales in twelve major keys and twelve minor keys and they were presented to participants in three different random orders. Participants were told to identify the key name and the mode. The results indicated that identification of these musical segments as being in major or as minor keys was not perfect, but that identification improved when the subjects were instructed to disregard key names and to judge only whether the key was major or minor. In general, major

\footnotetext{
*Acknowledgments: This research was supported in part by Grant-in-Aid for Scientific Research (C) (No. 15530473) from Japan Society for the Promotion of Science.

Sumi Shigeno, Ph.D., professor, Department of Psychology, Aoyama Gakuin University.
} 
keys were more often correctly identified than minor ones. He suggested that judgments of musical tonality are probably multi-dimensional and influenced by the set of stimuli. In Corso's experiment, musical notes from a diatonic scale were presented randomly and the melodies were written based on musical scales. Therefore, perhaps because participants could not draw on long term memory of melodies and keys in this task, these results indicate that the short-term memory is involved in the identification of musical key.

People who can remember and reproduce the names of musical notes without any reference tone are said to possess absolute pitch (AP) and it was reported that the AP ability is useful in recognizing melodies (Ward \& Burns, 1982). There have been various investigations of AP ability (e.g., Burns, Campbell, \& Hoberg, 1989; Siegel, 1974; Siegel \& Siegel, 1977a, 1977b; Burns \& Ward, 1978). Moreover, Gussmack, Vitouch, and Gula (2006) described that all-or-none models of absolute pitch are increasingly being replaced by a continuum view.

Musical intervals are described using frequency ratios of small integers. When two musical keys are called "related keys", their tonics are near in "harmonic distance” such as 1:2 (octave), 2:3 (fifth), and 3:4 (fourth), irrespective of their "linear distance" (i.e., pitch height offset). On the other hand, when two musical keys are called "remote keys", their tonics are far apart in "harmonic distance" (i.e., offset on the circle fifths), although they are near in "linear distance".

Terhardt and Ward (1982) explored about whether or not an individual's ability to identify tonality depends on possessing AP. They recorded the initial five passages of the twelve major-key preludes from "Well-Tempered Clavichord” by J. S. Bach. Participants were students of the music department of university. They judged whether each passage was played in the correct key (i.e., the original key), or in a key transposed either up or down. A simplified version of the original (correct) score was printed on the answer sheet. The results indicated that errors depended upon the "linear distance" between the original and its transposed key rather than the "harmonic distance" between them. This outcome suggested that a participant's ability to absolutely identify tonality (absolute tonality) is more widespread than commonly thought. The authors mentioned that there is a relationship between the ability to absolutely identify musical keys and AP possession. For example, a musically educated AP possessor should be able to identify any key by first recognizing some individual notes and then deducing the key on the basis of these recognized notes. This behavior may be common to all AP possessors.

In the procedure of Terhardt and Ward (1982), the participants identified the key after reading the score; therefore, AP possessors could identify the pitch of a note from the score as soon as they had heard the note. Accordingly, it seems likely that AP possessors can identify the pitch of each note, meaning that they could easily identify the key of melodies on the basis of their recalled pitch. In other words, the AP possessors didn't have to directly identify the key of a melody on the basis of key memory. In another experiment using 11 AP possessors and 124 non-AP (NAP) possessors, Terhardt and Seewann (1983) examined whether or not brief musical stimuli could be identified as being in the nominal (i.e., correct) key when no pitch reference was given. They suggested that there are two different modes of key identification: one used by NAP possessors and the other by AP possessors. They further suggested that no perceptual cues other than pitch are involved. They concluded that

both AP possessors and non-AP possessors depend on absolute pitch information when identifying musical key; however, they employ different perceptual modes: AP possessors primarily identify individual notes, while non-AP possessors unconsciously deduce from a series of notes a feeling of key. (Terhardt \& Seewann, p. 63) 
Vitouch and Gaugusch (2000) employed basically the same method with Terhardt and Ward (1982) using the nominal (original) key and digitally transposed to C\#, and tested the ability of high school students (amateur musicians) without AP to discriminate between these two keys. They found that they were significantly able to distinguish an original piece from even a one-semitone transposition. They proposed that also NAP possessors may have access to "latent" long-term pitch representations in auditory memory (e.g., for the pitches of well-known tunes) and that long-term memory for musical key in active and passive "absolute tonality" can be understood as weakened forms of AP.

The procedures adopted by Terhardt and Ward (1982) and Corso (1957) can be regarded as advantageous to AP possessors. The investigation on the perceptual mode of AP possessors is useful for exploring the information processing involved in identifying musical keys, and the results obtained from AP possessors have provided many important suggestions on key perception. However, the population of AP possessors is actually quite small (Corliss, 1973). It is necessary to explore the information processing of NAP possessors used in identifying musical keys. Consequently, any attempt to compare the identification process between AP and NAP possessors requires employment of procedures that are equally advantageous for both AP and NAP possessors.

Another point requiring clarification involves possible effects of pitch distance. In particular, the pitch distance of the tonic of a transposed key from pitch of the tonic of the original key (hereafter referred to as pitch range) may be relevant in this task. For instance, a judgment of musical key requires naming the transposed tonic in a particular passage, when it is higher or lower than the original key. However, in previous research, such as Terhardt and Ward (1982), these judgments were studied by varying the pitch distance of the transposed passage such that the farthest tonic from the original was higher or lower by seven semitones. This is problematic because the keynote that is higher or lower than the original by an octave (i.e., twelve semitones) should be included; this is because a key transposed higher or lower by an octave has the same nominal key as the original but a different pitch range. In other words, pitch range and key distance were confounded.

If we discuss which element of key or pitch range is essential in remembering/recalling a melody, it would be necessary to compare the memory of melodies in the two cases, in one of which the nominal key would be identical but pitch range different, and in the other case the nominal key would be different but pitch range small.

For these reasons, we required participants to rate their confidence in their memory of a melody depending upon how well they remembered it. Importantly this rating did not require participants to identify the keys. The impression of melodies might change as function of pitch range or if the pitch is altered to be higher or lower than the original piece because, as Shepard (1965) pointed out, when the pitch of a tone rises, its timbre is perceived to be sharper. This means that the impression of a melodic segment should be different if it is transposed higher or lower than the original key. Furthermore, as Corso (1957) noted, because the recognition process is multidimensional and responses would be made on the basis of both pitch perception and recognition of musical keys, the whole impression of a melody would be greatly changed by any transposition. In the current study, we employed several musical keys, including related keys and remote keys. We compared results between the original and transposed keys of a single musical piece, and at the same time the results between AP and NAP possessors.

Absolute tonality, if it exists, might be retained in memory as a series of absolute pitches, because both AP and NAP possessors depend on absolute pitch information when identifying musical keys (Terhardt \& Seewann, 1983). On the other hand, one might say that we don't remember melodies by the nominal key. If we don't remember melodies by the nominal key, in what way do we remember it? To clarify this issue it will be 
necessary to employ a method where both AP and NAP possessors have equal advantages. The current experiment required participants to rate the strength of their memory of melodies. This method might be superior to ones based on the identification of musical key, which were used in the previous research.

One purpose of the present study is to explore the relationship between memory and key transposition. A second goal is to investigate the relation between long-term memory strength and the judged familiarity of a musical piece.

\section{Method}

\section{Participants}

Eighty-one Japanese university students participated in partial return for course credit. All participants in an ordinary classroom reported that they had normal hearing. Prior to the experiment, all participants were tested to determine whether they actually possessed AP. Twenty recorded sounds played on a piano (DIAPASON, YAMAHA) were randomly presented one by one to the participants. Participants were required to identify the notes without comparing them with other tones. As a result, eight participants who absolutely identified (by name) more than 16 notes were selected as AP-possessors. One participant got full marks (20 scores), two each got 19 and 16 scores, and one got 18 scores (average $=18.0, \mathrm{SD}=1.5$ ). Most of the errors made by the AP-possessors were misidentification of the notes as being tones an octave higher or as lower than they actually were. The other 73 participants scored less than 13 correct and were judged as NAP possessors (average $=3.8, \mathrm{SD}=4.1$ ). The musical background of NAP participants was not known except the fact that they had learned western music at school for at least 12 years.

\section{Stimuli}

Synthetic grand-piano tones were produced by Music Creator (Roland, MR-NS20W). All tones were read out at a sampling rate of $44100 \mathrm{~Hz}$ with an accuracy of 16 bits. Several passages from two famous classical piano pieces were synthesized by the Music Creator: Für Elise by L.van Beethoven and Étude (op.10-3) by F. F. Chopin. The former was in the key of C-minor and the latter was in E-major. The total durations of the melodies were $25 \mathrm{~s}$ (Für Elise) and $39 \mathrm{~s}$ (Étude [op.10-3]).

Table 1 represents the seven keys used in the experiment.

Table 1

Music and Seven Keys Used for the Experiment

\begin{tabular}{llllllll}
\hline \multirow{2}{*}{ Tune } & \multicolumn{7}{c}{ Key } \\
\cline { 2 - 7 } & I & II & III & IV & V & VI & VII \\
\hline Für Elise & -12 & -7 & -1 & Original & +1 & +7 & +12 \\
Étude, op.10-3 & E major & A major & $\mathrm{D}^{\#}$ major & E major & F major & H major & E major \\
\hline
\end{tabular}

Notes. I: the key is lower than the original key by 12 semitones; II: the key is lower than the original key by 7 semitones; III: the key is lower than the original key by a semitone; IV: an original key; V: the key is higher than the original key by a semitone; VI: the key is higher than the original key by 7 semitones; VII: the key is higher than the original key by 12 semitones).

The original key was transposed as follows: (1) The same key as the original key but the pitch of its tonic was higher or lower than the tonic of the original key by an octave (twelve semitones). The linear distance from the original was great, but the harmonic distance was identical. (2) The key in which a tonic was higher or lower than the tonic of the original key by one semitone (remote keys). Here, the linear distance from the original was small but the harmonic distance was great. (3) The dominant or the subdominant key in which the 
tonic was higher or lower than the original key by seven semitones (related keys). The linear distance from the original was great, but the harmonic distance was small.

\section{Procedure}

Each melody was played in seven different keys, which were randomly selected and presented in counterbalanced order across different participants. Participants were required to rate the strength of their memory of the musical excerpt by comparing each excerpt with their long-term memory of it using a five-point scale (1.different-5.identical). At the end of each melody, participants were required to choose one of the three sentences on a three-point scale (1. I have heard this melody very often: defined as "high familiarity"; 2. I think I have ever heard this melody: "low familiarity"; 3. It is the first time that I have heard this melody: "unknown (no familiarity)").

The music was presented through loudspeakers at a comfortable listening level (about 56-69 dB A). The time allocated for judgment was about 10s in every trial.

\section{Results}

The current study aimed to examine long-term memory of musical pieces. Therefore, responses of the participants who reported no familiarity with the original piece were removed from the data as missing values in statistical analyses. Eleven participants did not know “Étude (op.10-3)".

Figure 1 shows the mean rating score averaged over participants. Rating scores are plotted as a function of the key (I VII). A three-way mixed-design analysis of variance (ANOVA) with 2 (participant: AP or NAP) x 7 (key: I, II, III, IV, V, VI, or VII) x 2 (excerpt: Für Elise or Étude [op.10-3]) yielded no significant interaction among the three factors. Only an interaction between participant and key was significant, $F(6,408)=2.24, p<$ 0.05. The effect of melody was not significant in main effect and in the interaction with key; therefore the responses obtained from the two melodies were merged in the following analyses. The relation between participant and key was compared. A two-way mixed-design ANOVA with 2 (participant: AP or NAP) x 7 (key: I, II, III, IV, V, VI, or VII) yielded a significant interaction, $F(6,408)=2.24, p<0.05$. Simple main effects of key were significant: in the case of AP-possessors, $F(6,408)=14.60, p<0.001$, in the case of NAP-possessors, $F(6,408)=68.22, p<0.001$. The results of multiple comparisons by Bonferroni are shown in Table 2. Figure 1 and Table 2 show that, in both AP and NAP, the memory ratings were highest for the original key, but as the key transposition increased in linear distance from the original, the memory ratings declined except in the case of the transposition higher or lower by an octave (twelve semitones). Simple main effects of participant were not significant except in the case of key $\mathrm{V}$ (higher than the original by one semitone), $F(1,68)=7.05, p<0.05$.

(a) Für Elise

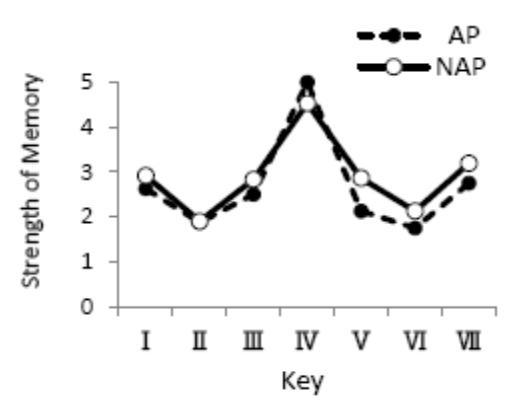

(b) Étude, op.10-3

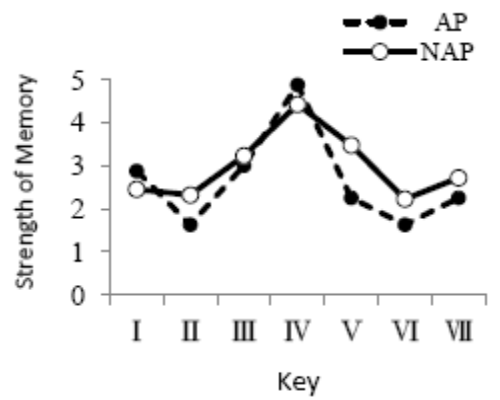

Figure 1. Strength of memory to recognize the melodies. 
Table 2

The Results of Multiple Comparisons by Bonferroni Between Seven Keys

\begin{tabular}{|c|c|c|c|c|c|c|c|}
\hline \multicolumn{8}{|c|}{ (a) AP-possessors } \\
\hline Key & I & II & III & IV (original) & $\mathrm{V}$ & VI & VII \\
\hline I & - & & & & & & \\
\hline II & * & - & & & & & \\
\hline III & & *** & - & & & & \\
\hline IV & $* * *$ & *** & $* * *$ & - & & & \\
\hline $\mathrm{V}$ & & & & *** & - & & \\
\hline VI & * & & $* *$ & $* * *$ & & - & \\
\hline VII & & & & *** & & * & - \\
\hline \multicolumn{8}{|c|}{ (b) NAP-possessors } \\
\hline Key & I & II & III & IV (original) & $\mathrm{V}$ & VI & VII \\
\hline I & - & & & & & & \\
\hline II & $* *$ & - & & & & & \\
\hline III & $*$ & $* * *$ & - & & & & \\
\hline IV & $* * *$ & $* * *$ & $* * *$ & - & & & \\
\hline $\mathrm{V}$ & $* *$ & $* * *$ & & $* * *$ & - & & \\
\hline VI & $* *$ & & *** & $* * *$ & $* * *$ & - & \\
\hline VII & * & 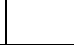 & **** & $* * *$ & & * & - \\
\hline
\end{tabular}

Figure 2 shows relationships between the declared familiarity of melodies and memory ratings. Participants categorized into the high-familiarity and the low-familiarity groups for "Für Elise" were 53 and 20, respectively; the high-familiarity group and low-familiarity group for "Étude (op.10-3)" were 14 and 39, respectively. The results for both melodies were very similar. A two-way ANOVA with 2 (high familiarity vs. low familiarity) $\times 7$ (key: I - VII) was conducted for each melody. The results showed that there was no significant interaction and no main effect of strength of memory. The main effect of key was significant; Für Elise, $F(6,426)=35.42, p<0.001$; Étude (op.10-3), $F(6,360)=40.70, p<0.001$. The results mean that the strength of familiarity of the melodies has nothing to do with the relationship between the strength of memory and keys.

(a) Für Elise

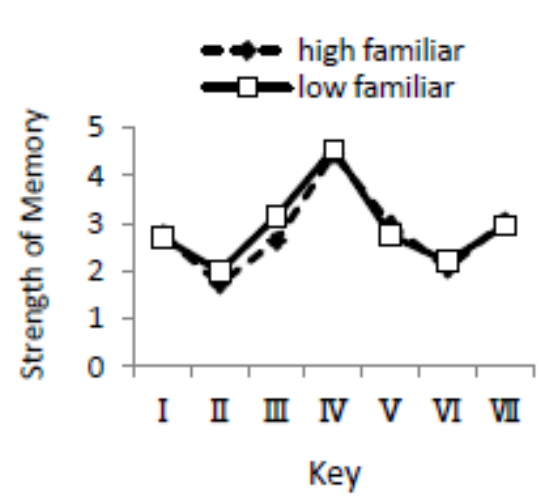

(b) Étude, op.10-3

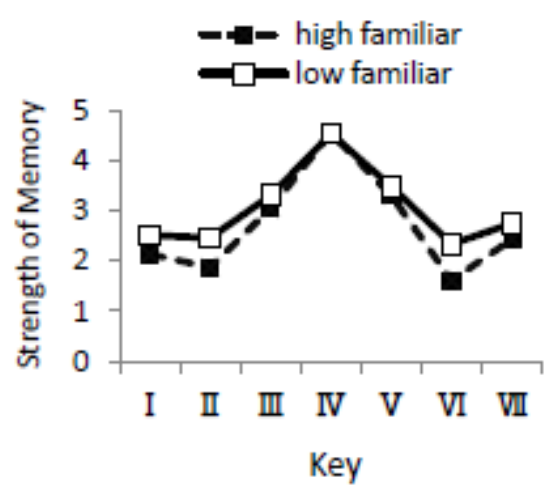

Figure 2. The relation between the strength of memory and the familiarity of the melodies. 


\section{Discussion}

The current research examined memory strength and transposed keys, and the relation between the memory strength and the judged familiarity of a musical piece. A comparison between AP and NAP possessors yielded several important findings: (1) Both AP and NAP possessors memorized melodies mainly by means of pitch range, and partially by the musical keys. (2) Memory of melodies was strongest for those pieces presented in their original key, but the further the key was transposed from the original in linear distance, the more memory declined. An exception was the case of a transposition by an octave. (3) Familiarity of melodies had no effect on the relationship between the strength of memory and keys.

The current results suggest that both AP and NAP possessors remember melodies by means of pitch range rather than by means of musical key. That is, when a key was transposed higher or lower by an octave, memory ratings were not as high as they were for the original melody. Furthermore, the fact that the familiarity of melodies had little effect on the relationship between the strength of memory and keys, suggests that the memory of pitch range of melodies is robust.

Figure 3 shows three hypothetical models that could qualitatively describe the performance for familiar melodies. The abscissa represents the key transposed from the original key. An original key is positioned at the middle of the abscissa. The ordinate represents the strength of memory. Model (a) represents the relationship between the strengths of memory for melodies and keys, if a listener has no absolute tonality s/he would then remember melodies based only on the pitch range. As a result, the highest scores would be given to the original key and lowest scores to transposed octaves. Thus, the scores would be plotted as a function of the "linear distance" from the original key. Model (b) shows the relationship between the memory strength for melodies and keys, if a listener has absolute tonality. The listener remembers the melodies only from their tonality (musical key). As a result, the highest scores would be given to both the original and to the keys transposed higher or lower by an octave, and lower scores would be given to other keys. The scores would be dependent on the "harmonic distance". Model (c) is the case where a listener remembers melodies by both pitch range and absolute musical key, although pitch range has greater weight. In this case, the graph forms a shape that is nearly the average between model (a) and model (b). Model (c) fits best with the current results.
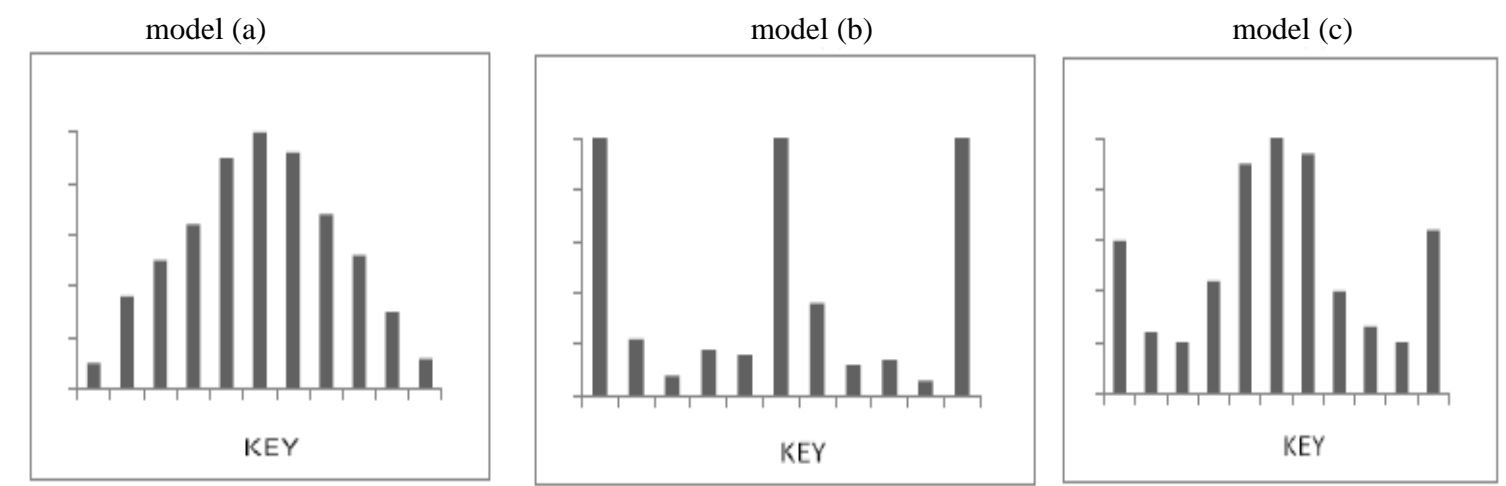

Figure 3. Three hypotheses for the memory of familiar melodies. Model (a) shows that the results would be obtained if a listener has memorized familiar melodies by means of pitch range and not by key. Model (b) shows that the results would be obtained if participants had memorized familiar melodies by means of absolute tonality and not by pitch range. Model (c) shows that the results would be obtained if participants remembered familiar melodies by both pitch range and absolute tonality. The abscissa represents the distance from the original key (middle of the abscissa) to the transposed keys. The transposed keys are \pm 1 semitone (remote keys), \pm 7 semitones (related keys), and \pm 12 semitones (octave) from the original. The ordinate represents the strength of memory. 
If we listen to melodies played higher than the original by one octave, we would feel these musical pieces to be different from the original, even if the musical key is the same as the original key. It appears that absolute tonality is quite difficult to prove. The current results strongly indicate that memory of music depends on both pitch range and musical key, although the former is, for our long-term memory, more essential and influential than the latter.

\section{Conclusion}

The current research investigated the relationship between memory of melodies and musical keys. Whether our memory of music is based on absolute tonality has been the subject of academic discussion, and most experiments exploring this problem have employed a key identification method. However, AP possessors are superior to NAP in key identification. In the present experiment, instead of a key identification method, participants were asked about their strength of memory. As a result, it was found that both AP and NAP possessors remembered melodies mainly via the pitch range of the melodies, and that the memory of melodies was best in the original key. It was further found that familiarity of music has no effect on the relationship between the strength of memory and keys. Three hypotheses for the long-term memory of melodies were presented. Future research with other genre of music will be necessary to confirm the current findings.

\section{References}

Burns, E. M., \& Ward, W. D. (1978). Categorical perception-Phenomenon or epiphenomenon: Evidence from experiments in the perception of melodic musical intervals. Journal of the Acoustical Society of America, 63, 456-468.

Burns, E. M., Campbell, K. S., \& Hoberg, K. E. (1989). Is absolute pitch an example of “categorical perception”? Proceedings of the First International Conference on Music Perception and Cognition (pp. 421-424).

Butler, D., \& Brown, H. (1994). Describing the mental representation of tonality in music. In R. Aiello (Ed.), Musical perceptions (pp. 191-212), Oxford: Oxford University Press, Inc.

Corliss, E. L. R. (1973). Remark on “Fixed-scale mechanism of absolute pitch”. Journal of the Acoustical Society of America, 53, $1737-1739$.

Corso, J. F. (1957). Absolute judgments of musical tonality. Journal of the Acoustical Society of America, 29, $138-144$.

Gussmack, M. B., Vitouch, O., \& Gula, B. (2006). Latent absolute pitch: An ordinary ability? In M. Baroni, A. R. Addessi, R. Caterina, \& M. Costa (Eds.), Proceedings of the 9th International Conference on Music Perception and Cognition (pp. 1408-1412). Bologna, Italy: Bononia University Press.

Marvin, E. W. (1997). Tonal/Atonal: Cognitive strategies for recognition of transposed melodies. In D. Baker, D. Beach, \& J. Bernard (Eds.), Music theory in concept and practice (pp. 217-236). Rochester: University of Rochester Press.

Shepard, R. N. (1965). Representation of structure in similarity data: Problems and prospects. In D. I. Mostofsky (Ed.), Stimulus generalization (pp. 94-110). Stanford: Stanford University Press.

Siegel, J. A. (1974). Sensory and verbal coding strategies in subjects with absolute pitch. Journal of the Experimental Psychology, 103, 37-44.

Siegel, J. A., \& Siegel, W. (1977a). Absolute identification of notes and intervals by musicians. Perception \& Psychophysics, 21, 143-152.

Siegel, J. A., \& Siegel, W. (1977b). Categorical perception of tonal intervals: Musicians can’t tell sharp from flat. Perception \& Psychophysics, 21, 399-407.

Temperley, D., \& Marvin, E. W. (2008). Pitch-class distribution and the identification of key. Music Perception, 25, $193-212$.

Terhardt, E., \& Seewann, M. (1983). Aural key identification and its relationship to absolute pitch. Music Perception, 1, 63-83.

Terhardt, E., \& Ward, W. D. (1982). Recognition of musical key: Exploratory study. Journal of the Acoustical Society of America, 72, 26-33.

Thompson, W. F., \& Robitaille, B. (1992). Can composers express emotions through music? Empirical Studies of the Arts, 10, 79-89. 
Vitouch, O., \& Gaugusch, A. (2000). Absolute recognition of musical keys in non-absolute-pitch-possessors. In C. Woods, G. Luck, R. Brochard, F. Seddon, \& J. A. Sloboda (Eds.), Proceedings of the 6th International Conference on Music Perception and Cognition [CD-ROM]. Keele, UK: Dept. of Psychology, Keele University.

Ward, W. D., \& Burns, E. M. (1982). Absolute pitch. In D. Deutsch (Ed.), The psychology of music (pp. 431-451). New York: Academic Press. 